Revista de Psicología de la PUCP. Vol. XIX, 1, 2001

\title{
Autoconcepto y desesperanza aprendida en un grupo de maestros de Lima Metropolitana
}

\author{
Sandra Carrillo Luna' \\ Pontificia Universidad Católica del Perú
}

\begin{abstract}
Se evaluó a ochenta y seis maestros de Lima capacitados por el PLANCAD con el Perfil de Autopercepción para Adultos (Messer y Harter, 1986) y con la Escala de Desesperanza Aprendida (Macassi, 1988). Se analizó las correlaciones entre los distintos dominios del autoconcepto (AC), la desesperanza aprendida (DA) y algunas variables sociodemográficas. Los resultados mostraron que la DA presenta correlaciones significativas e inversas con los dominios del AC de inteligencia, competencia laboral (CL), sociabilidad, adecuación como proveedor de apoyo (APA), apariencia física (AF) y relaciones cercanas (RC); así como con el rendimiento académico y grado de satisfacción con el trabajo. Se presentaron correlaciones significativas de la edad con los dominios del AC de sociabilidad y RC; satisfacción con la carrera, con los dominios del AC de sociabilidad, APA, CL y $\mathrm{RC}$; satisfacción con el trabajo, con los dominios del AC de inteligencia, administración del hogar, CL, APA, AF y RC; y el rendimiento académico, con el dominio de sociabilidad. Asimismo, se presentaron diferencias significativas entre los puntajes obtenidos según sexo, institución en la que terminaron sus estudios y si han recibido o no otras capacitaciones.
\end{abstract}

Palabras clave: autoconcepto, desesperanza aprendida, maestros, PLANCAD.

Self Concept and Learned Helplessness in a group of teachers from Lima Metropolitana 86 teachers from Lima Metropolitana trained by PLANCAD were assessed with the Self Perception Profile for Adults (Messer y Harter, 1986) and with a Learned Helplessness Scale (Macassi, 1988). Correlations between the different domains from Self Concept (SC), Learned Helplessness (LH) and some social-demographic variables were analyzed. The results showed that the LH exhibits significant and inverse correlations with some domains of SC such as: Intelligence, Job Competence (JC). Sociability, Adequate Provider (AP), Physical Appearance (PA), Intimate Relationships (IR), Academic Achievement (AA) and the Job Satisfaction Degree. They also showed significant correlations between the variable age and two domains of SC: Sociability and IR; between Career Satisfaction and the SC domains of Sociability, AP, JC and IR; also between Job Satisfaction and the SC domains of Intelligence, Household Management, JC, AP, PA and IR. Finally significant correlations were showed between Academic Achievement with the domain of Sociability. The results also showed significant differences between the punctuation obtained from the variables of sex, the institutions were they finished their education and the variable have/have not received other qualifications.

Keywords: self concept, learned helplessness, teachers, PLANCAD.

1. Licenciada en Psicología Educacional de la Pontificia Universidad Católica del Perú. Trabajó en el PLANCAD, en el área de Evaluación y Monitoreo. Actualmente se desempeña en el proyecto PLANCAD GTZ KFW de la Cooperación Técnica Alemana que asesora a la Unidad de Capacitación Docente del Ministerio de Educación. Asimismo se desempeña como jefe de práctica en la PUCP. 

Si se toma en consideración la compleja y difícil realidad en la que viven y trabajan los maestros del Estado en la actualidad, es importante saber si estos, enfrentando condiciones adversas de trabajo, han desarrollado o no la desesperanza aprendida. Estudiar la relación de esta con el autoconcepto y, sobre todo, conocer al docente en cuanto a cómo él mismo se concibe puede ser una base importante para enfocar la capacitación y la profesionalización de una manera más directa y concreta, de tal manera que estas acciones tengan resultados positivos y se conviertan en una motivación constante tanto para mejorar como para saber que la repercusión de estas tendrá efectos positivos en la sociedad futura.

En la actualidad, el autoconcepto cobra importancia debido a la influencia que tiene en los diferentes aspectos de la vida. Arul (1972) sostiene que este juega un rol vital en el desarrollo de la personalidad: la manera cómo se percibe el individuo va perfilando sus patrones de comportamiento; en este sentido, las personas actúan de manera consistente a la forma cómo se ven ellos mismos, es decir, a su autoimagen. Markus y colaboradores (Harter, 1999) estudian los procesos del yo en adultos y han centrado su estudio en cómo el yo organiza, interpreta y da significado a las experiencias; regula el afecto y motiva la acción dando incentivos, estándares, planes y guiones. Por otro lado, la construcción de los yo posibles en el futuro organiza el comportamiento y energetiza al individuo para seguir metas seleccionadas. Las discrepancias entre el autoconcepto real e ideal pueden también motivar al individuo a conseguir sus ideales en servicio de la mejora del yo.

Cuando una persona enfrenta situaciones donde los resultados le son incontrolables, aprende que sus respuestas y los resultados son independientes los unos de los otros. La atribución que el sujeto hace referente a esa falta de control experimentada puede generar la ex- 
pectativa de una falta de control de sus respuestas sobre los resultados. Esta expectativa es la que determina la generalidad, duración y características de los efectos conductuales de la desesperanza (Castrillo, 1986). La situación material de la falta de control, la percepción de no tener control sobre el medio, la atribución sobre la causa que originó la consistencia actor-modificación, la expectativa de no tener control sobre el futuro y los comportamientos producidos son los cinco momentos que generan la desesperanza aprendida (Macassi, 1988).

Desde 1995, el Ministerio de Educación inició un proceso de modernización del sistema educativo denominado Programa Especial de Mejoramiento de la Calidad de la Educación Peruana (MECEP) en el que contempla diferentes aspectos, como plantear un nuevo programa curricular, implementar materiales educativos para el trabajo en el aula, mejorar la infraestructura de los centros educativos, capacitar a los directores en aspectos de gestión, mejorar la calidad de la formación docente y capacitar a los maestros para optimizar su trabajo en el aula, entre otros. El subcomponente de Capacitación Docente (PLANCAD), que es parte del Programa MECEP, ha considerado que para elevar el nivel de los distintos factores educativos y que estos sean sostenibles en el tiempo, el docente debe ser considerado como principal gestor, agente y guía de todo proceso educativo. Es dentro de este marco que se han estructurado una serie de acciones, organizadas en un programa, que se han venido desarrollando desde 1995 con el objetivo de ayudar a solucionar las carencias de métodos y técnicas que faciliten la tarea docente en el aula (Ministerio de Educación, 1999).

La relación que existe entre la concepción que el docente tiene sobre sí mismo y las atribuciones y conductas frente a determinadas situaciones en su trabajo son una parte importante y complementaria a lo que está planteando el Ministerio de Educación en la actualidad con relación a la capacitación y formación docente. Esta concepción puede tener una relación directa con el hecho de cómo se desempeña en la capacitación y en su formación. El autoconcepto del docente 
debe ser un factor que favorezca el proceso educativo en la dirección deseada por lo que puede ser tomado como referencia para la formulación de estrategias necesarias y así lograr una mayor efectividad en el cambio.

Pregunta de investigación

A partir de lo anteriormente expuesto, este estudio se propuso responder las siguientes interrogantes: ¿cómo es el autoconcepto en un grupo de maestros que reciben capacitación por parte del programa PLANCAD del Ministerio de Educación? ¿Existe desesperanza aprendida en estos maestros? Y si existiera, ¿en qué medida el autoconcepto de los maestros se relaciona con la desesperanza aprendida?

Es así que la presente investigación tuvo como objetivo básico describir el autoconcepto en los maestros de primaria, capacitados por el PLANCAD, en los diversos dominios: inteligencia, competencia en el trabajo, competencia atlética, apariencia física, sociabilidad, relaciones cercanas, moralidad, sentido del humor, crianza, administración del hogar, adecuación como proveedor de apoyo, así como autovaloración global o autoestima según la Escala de Autopercepción para Adultos de Messer y Harter (1986).

Las hipótesis de investigación planteadas fueron las siguientes:

Hipótesis general:

Existen correlaciones inversas entre los distintos dominios del autoconcepto y la desesperanza aprendida en los maestros capacitados por el PLANCAD.

Hipótesis específicas:

- Existen correlaciones entre los distintos dominios del autoconcepto y las variables edad, grado de satisfacción con la carrera 
estudiada, grado de satisfacción con el trabajo que realiza como docente y el rendimiento académico en el taller de capacitación.

- Existen diferencias significativas entre los distintos dominios del autoconcepto según las variables sexo, institución en la que el docente terminó sus estudios y otras capacitaciones recibidas además del PLANCAD.

- Existe una correlación inversa entre la desesperanza aprendida y el rendimiento académico en el taller de capacitación, así como con el grado de satisfacción con la carrera estudiada y el grado de satisfacción con el trabajo que realiza como docente.

\section{Metodología}

\section{Participantes}

Se trabajó con 86 maestros de escuelas primarias de Lima Metropolitana que estaban siendo capacitados por el PLANCAD en el año 2000 y que durante la investigación laboraban en centros educativos con polidocencia completa (un profesor por grado). Ellos fueron elegidos a través de un muestreo multietápico, no probabilístico intencional.

Las etapas de selección fueron las siguientes:

1. Selección de las USEs (Unidad de Servicios Educativos), que agrupaban a un porcentaje mayor al $13 \%$ de los docentes y $15 \%$ de los centros educativos de Lima.

2. Selección de un distrito dentro de cada USE escogida (distrito con mayor número de maestros y centros educativos dentro de cada USE escogida).

3. Ubicación de entes ejecutores y talleres de cada distrito (se ubicaron a las instituciones responsables (entes ejecutores) de la capacitación del PLANCAD en estos distritos con el fin de contactar a los maestros en los talleres de capacitación). 
4. Selección de maestros en cada taller (en estos talleres se escogieron accidentalmente docentes de diferentes grados de primaria que cumplieran con el requisito de trabajar en centros educativos de los distritos previamente seleccionados).

En el Cuadro 1 se presenta la conformación de los participantes, de acuerdo con la USE y con el distrito de donde provenían.

\section{Cuadro 1}

Distribución de la muestra según USE y distritos

\begin{tabular}{|cll|}
\hline USE & \multicolumn{1}{c|}{ Distrito } & $n$ \\
\hline 01 & San Juan de Miraflores & 33 \\
02 & San Martín de Porres & 33 \\
04 & Comas & 20 \\
Total & & 86 \\
\hline
\end{tabular}

\section{Instrumentos}

1. Perfil de Autopercepción para Adultos (Messer y Harter, 1986). Escala politómica de medición ordinal para adultos entre los 20 y 55 años, conformada por cincuenta ítems que se agrupan en doce dominios de competencia, cada uno de los cuales constituye una subescala separada:

- Sociabilidad: comportamiento de uno mismo ante la presencia de otros.

- Competencia en el trabajo: percepciones que se tienen sobre las competencias ocupacionales de uno mismo para el trabajo al que se le dedica mayor tiempo.

- Crianza: proceso de cuidar a otros.

- Competencia atlética: concepto que uno tiene sobre las habilidades para los deportes.

- Apariencia física: percepción de la manera como uno luce.

- Adecuación como proveedor de apoyo: percepción de proveer 
los medios de soporte para uno mismo y para los otros que son significativos.

- Moralidad: percepción del comportamiento basado en los estándares de conducta y de lo que es correcto o incorrecto.

- Administración del hogar: guiar o manejar actividades para el mantenimiento del hogar.

- Relaciones cercanas: implican acercamiento, interacciones significativas o relación entre el(a) compañero(a), la pareja y/ o una amistad especial.

- Inteligencia: habilidad para aprender y conocer.

- Sentido del humor: habilidad para ver el lado divertido de las cosas.

- Autovaloración global: percepción global de lo que uno vale como persona, independientemente de cualquier dominio particular o competencia. Esta escala es independiente de cualquier otro dominio y no constituye la suma de los puntajes de las otra subescalas.

El instrumento fue traducido y validado (Carrillo, 2000) por siete jueces con el criterio de índice de acuerdos (Escurra, 1998); se obtuvo porcentajes de acuerdo desde un $86 \%$ hasta $100 \%$. Se realizó una doble traducción del inglés al castellano y vuelta al inglés para lograr una versión definitiva en castellano que asegurara el sentido original. Un juez bilingüe revisó esa versión final y la comparó con la versión original en inglés y realizó las correcciones pertinentes. Luego se administró la prueba a un grupo piloto formado por diez maestros, en actividad, de los distritos seleccionados. A partir de este piloto se recogieron sugerencias para frasear algunos ítems (por ejemplo, utilizar la frase "relaciones cercanas" en vez de "relaciones íntimas"), se redactaron las instrucciones en una página aparte y se pusieron ejemplos, además de utilizar un papelógrafo para la explicación.

El instrumento, en su versión original, presentó coeficientes de confiabilidad de 0.63 a 0.92 . En nuestro medio, la confiabilidad de las subescalas se realizó a través del Alpha de Cronbach que presentó 
coeficientes de 0.21 a 0.75 . A pesar de los niveles de confiabilidad en los dominios de sentido del humor y crianza, no se eliminó ningún ítem para poder obtener los puntajes de las subescalas según lo planteado en la versión original. Para el análisis solo se utilizaron los dominios que demostraron un nivel de confiabilidad aceptable (mayor que .5). Se tomó la decisión de mantener los dominios originales porque el tamaño de la muestra resultaba ser insuficiente para el análisis factorial.

2. Escala sobre Desesperanza Aprendida (Macassi, 1988). Escala tipo Likert de doce ítems agrupados originalmente en tres áreas: falta de motivación, falta de control y expectativa de no control en el futuro. Esta escala fue construida sobre la base de entrevistas y observaciones a la población a la cual estaba dirigida (obreros de una pequeña empresa de Lima Metropolitana) y validada en un estudio piloto.

Para este estudio se realizaron adaptaciones en la redacción de los ítems que estaban referidos a una población obrera (Carrillo, 2000). Se cambiaron términos como "trabajo vecinal", "tareas vecinales sindicales", "gente del vecindario" y "problemas del vecindario" por "tareas educativas", "personas de la escuela" y "problemas de la escuela", respectivamente. Estos términos estaban el área de falta de motivación.

La escala, en su versión original, obtuvo un Alpha de Cronbach de 0.53 que indica una confiabilidad media o aceptable. Del total de la escala no se tuvieron en cuenta los ítems 3, 6 y 11 por no ser discriminativos. Para efectos de la presente investigación, la confiabilidad de las subescalas se realizó a través del Alpha de Cronbach. El análisis de confiabilidad arrojó un alpha de 0.52 para la escala total luego de haber eliminado los ítems $1,2,6,8$ y 11 que evidenciaron pobre validez de constructo. Debido a la confiabilidad media que arrojó la prueba y lo resultados del análisis factorial, que no resultó en una definición de áreas coherentes, 
no se tuvo en cuenta para el análisis los puntajes por áreas. La desesperanza aprendida se definió como el puntaje total de los siete ítems válidos siguiendo el procedimiento planteado inicialmente por el autor, 14 puntos indicarían una alta desesperanza aprendida.

3. Ficha de Datos Personales. Elaborada para los fines de esta investigación (Carrillo, 2000). Recoge información sobre los docentes como sexo, edad, lugar de nacimiento, tiempo de residencia en Lima (si nació en provincias), situación laboral (nombrado o contratado), ciclo o grado que enseña, tiempo de servicios en el Estado, institución en la que terminó sus estudios, grado de satisfacción con la carrera estudiada y el trabajo que realiza como docente, así como otras capacitaciones pedagógicas recibidas además del PLANCAD.

4. Rendimiento Académico. Se refiere al desempeño académico del docente obtenido durante el segundo taller de capacitación expresado en las calificaciones (en puntaje vigesimal) otorgadas por el ente ejecutor que lo capacita según los indicadores planteados por el PLANCAD.

\section{Resultados}

Respecto de los doce dominios evaluados a través del Perfil de Autopercepción para Adultos (Messer y Harter, 1986), los maestros presentaron más o menos una misma distribución en los puntajes sobre un rango posible para todos los dominios que oscila entre 1 y 4 puntos (véase Cuadro 2). 
Autoconcepto y desesperanza aprendida en un grupo de maestros de Lima Metropolitana

Cuadro 2

Percepción de los doce dominios del autoconcepto en los docentes

\begin{tabular}{|lcl|}
\hline Dominios del autoconcepto & Promedio & $D E$ \\
\hline Crianza & 3.65 & 0.42 \\
Inteligencia & 3.42 & 0.52 \\
Autovaloración global & 3.27 & 0.58 \\
Administración del hogar & 3.25 & 0.72 \\
Moralidad & 3.25 & 0.68 \\
Sociabilidad & 3.18 & 0.60 \\
Adecuación como proveedor de apoyo & 3.15 & 0.66 \\
Competencia laboral & 3.14 & 0.65 \\
Apariencia física & 3.09 & 0.71 \\
Relaciones cercanas & 3.08 & 0.69 \\
Sentido del humor & 2.75 & 0.61 \\
Competencia atlética & 2.66 & 0.72 \\
\hline
\end{tabular}

Los maestros evaluados han obtenido medias superiores al puntaje 2 en cada dominio. Esto los ubica por encima de un puntaje medio, en una escala de 4 puntos, presentándose un sesgo positivo en la percepción de su autoconcepto respecto de estos dominios. Sin embargo, los dominios de crianza y sentido del humor no entraron en el análisis por presentar ítems con baja consistencia interna.

Los dominios de inteligencia y autovaloración global son los que presentan los mayores promedios. Los dominios con mayor dispersión en los puntajes son los referidos a la administración del hogar y la apariencia física, así como competencia atlética, que además es el dominio que presenta el promedio más bajo.

Respecto de la distribución de la muestra para cada ítem en la Escala de Desesperanza Aprendida sobre un rango posible para todos los ítems que oscila entre 0 y 2 puntos ( 14 para el puntaje total), se observa que el puntaje total obtenido está por debajo de un puntaje medio, lo que indica que se presenta un bajo nivel de desesperanza aprendida en los maestros. Asimismo, se observa una distribución homogénea en las respuestas (véase Cuadro 3). 


\section{Cuadro 3}

Promedios de respuesta y puntaje total en la Escala de Desesperanza Aprendida

\begin{tabular}{|c|c|c|c|}
\hline $\mathrm{N}^{\circ}$ & Ítem & Promedio & $D E$ \\
\hline & El futuro es difícil de asegurar pues no se sabe que va pasar & 1.08 & 0.97 \\
\hline & No me conviene reclamar mucho pues no se consigue nada & & \\
\hline & más que nuevos problemas & 0.41 & 0.76 \\
\hline \multicolumn{3}{|c|}{ 5. Todos debemos conformarnos con lo que tenemos pues es } & 0.37 \\
\hline \multicolumn{4}{|c|}{ 7. En la mayoría de los casos los problemas no de dependen } \\
\hline & de uno mismo, sino de la difícil situación que uno vive & 1.21 & 0.95 \\
\hline \multicolumn{4}{|c|}{ 9. Es muy poco lo que se puede hacer para evitar nuevos } \\
\hline \multicolumn{4}{|c|}{ 10. Por lo general suceden más cosas malas que buenas en la } \\
\hline & vida y eso no depende de nosotros & 0.56 & 0.88 \\
\hline & Creo que los problemas de mi escuela no podrán solucionarse & 0.07 & 0.34 \\
\hline & Puntaje Total & 3.63 & 2.59 \\
\hline
\end{tabular}

En el análisis según el tipo de respuestas, en el ítem 3 ("El futuro es difícil de asegurar pues no se sabe que va pasar") y en el ítem 7 ("En la mayoría de los casos los problemas no de dependen de uno mismo, sino de la difícil situación que uno vive), se observa una cierta polarización en ellas ya que la mitad de los maestros están de acuerdo con dichas afirmaciones y la otra mitad en desacuerdo. Por esta razón la distribución en ambọs ítems es mayor que el resto.

Asimismo, se observa que la menor distribución se presenta en los ítems 5 ("Todos debemos conformarnos con lo que tenemos pues es muy difícil de conseguir mayores ingresos económicos") y 12 ("Creo que los problemas de mi escuela no podrán solucionarse") en donde casi todos los participantes están en desacuerdo con dichas afirmaciones.

En el Cuadro 4 se presenta la correlación entre los diversos dominios del autoconcepto y algunas variables. 
Autoconcepto y desesperanza aprendida en un grupo de maestros de Lima Metropolitana

\section{Cuadro 4}

Correlación Pearson entre los dominios del autoconcepto y algunas variables

\begin{tabular}{|lccccc|}
\hline Dominios del Autoconcepto & $\begin{array}{c}\text { Desesperanza } \\
\text { Aprendida }\end{array}$ & Edad & $\begin{array}{c}r \\
\text { Satisfacción } \\
\text { Con Carrera }\end{array}$ & $\begin{array}{c}\text { Satisfacción } \\
\text { con Trabajo }\end{array}$ & $\begin{array}{c}\text { Rendimiento } \\
\text { Académico }\end{array}$ \\
\hline Inteligencia & $-0.29^{* *}$ & 0.03 & 0.18 & $0.29^{* *}$ & 0.14 \\
Autovaloración global & -0.21 & -0.13 & 0.07 & 0.07 & 0.12 \\
Administración del hogar & -0.05 & 0.05 & 0.13 & $0.24^{*}$ & 0.09 \\
Moralidad & -0.20 & 0.03 & 0.02 & 0.07 & 0.20 \\
Sociabilidad & $-0.23^{*}$ & $0.25^{*}$ & $0.24^{*}$ & 0.13 & $0.23 *$ \\
Adecuación como proveedor de apoyo & $-0.24^{*}$ & -0.12 & $0.40^{* *}$ & $0.24^{*}$ & 0.16 \\
Competencia laboral & $-0.29^{* *}$ & -0.08 & $0.36^{* *}$ & $0.41^{* *}$ & 0.17 \\
Apariencia física & $-0.23^{*}$ & 0.01 & 0.14 & $0.26^{*}$ & 0.02 \\
Relaciones cercanas & $-0.22-$ & $0.28^{*}$ & $0.27^{*}$ & $0.22^{*}$ & 0.21 \\
Competencia atlética & -0.03 & -0.09 & -0.05 & 0.02 & -0.06 \\
\hline
\end{tabular}

Nota. ${ }^{*} p<.05$

$* * p<.01$

La desesperanza aprendida guarda una relación inversa con todos los puntajes de los dominios de autoconcepto, lo que quiere decir que a menor desesperanza aprendida mayor percepción de uno mismo en estos dominios y viceversa. La correlación de la desesperanza aprendida es estadísticamente significativa solo en los dominios sociabilidad, adecuación como proveedor de apoyo, apariencia física y relaciones cercanas $(p<.05)$; mientras que a un nivel de significancia mayor $(p<.01)$ se correlaciona significativamente con los dominios de competencia laboral e inteligencia.

Los dominios de sociabilidad y relaciones cercanas correlaciona con la edad a un nivel significativo $(p<.05)$, lo que indica que a mayor edad mayor percepción en estos dominios y viceversa. De la misma manera, se presentan correlaciones no significativas, pero inversas con los dominios de autovaloración global, adecuación como proveedor de apoyo, competencia laboral y competencia atlética.

El grado de satisfacción con la carrera estudiada correlaciona con los dominios de sociabilidad y relaciones cercanas a un nivel significativo $(p<.05)$, mientras que los dominios de competencia laboral y 
adecuación como proveedor de apoyo correlacionan a un nivel de significancia mayor $(p<.01)$. Es importante señalar que el dominio de competencia atlética correlaciona de manera inversa con el grado de satisfacción de la carrera estudiada, aunque no de una manera significativa.

El grado de satisfacción con el trabajo que realizan los docentes correlaciona significativamente con los dominios de administración del hogar, adecuación como proveedor de apoyo, apariencia física y relaciones cercanas $(p<.05)$ y con inteligencia y competencia laboral a un nivel mayor de significancia $(p<.01)$.

El rendimiento académico correlaciona significativamente $(p<$ $.05)$ con el dominio de sociabilidad. En los demás no se presentan relaciones significativas. Asimismo, se presenta una relación inversa con el dominio de competencia atlética, aunque esta no es significativa.

En el Cuadro 5 se presenta los resultados del análisis de las diferencias entre los dominios del autoconcepto de acuerdo con las características de la muestra.

\section{Cuadro 5}

Diferencia de medias entre los dominios del autoconcepto y algunas variables

\begin{tabular}{|c|c|c|c|c|c|}
\hline Dominios del autoconcepto & $\begin{array}{l}\text { Sexo } \\
\text { (F-M) }\end{array}$ & $\begin{array}{l}\text { Institución } \\
\text { (U- I) }\end{array}$ & $\begin{array}{c}\text { Capacitaciones } \\
\text { (S-N) }\end{array}$ & $\begin{array}{l}\text { Capacitacione } \\
\text { MED (S-N) }\end{array}$ & $\begin{array}{l}\text { es Capacitaciones } \\
\text { No MED (S-N) }\end{array}$ \\
\hline Inteligencia & -0.10 & -0.27 & -0.10 & -0.08 & 0.34 \\
\hline Autovaloración global & 0.33 & 0.00 & 0.18 & 0.04 & 0.38 \\
\hline Administración del hogar & -0.36 & -0.02 & 0.14 & -0.06 & -0.15 \\
\hline Moralidad & 0.34 & -0.23 & 0.00 & -0.11 & 0.36 \\
\hline Sociabilidad & -0.32 & -0.25 & 0.09 & -0.09 & 0.21 \\
\hline $\begin{array}{l}\text { Adecuación como } \\
\text { proveedor de apoyo }\end{array}$ & 0.04 & -0.15 & 026 & 028 & 0.22 \\
\hline Competencia laboral & -0.06 & -0.28 & $\begin{array}{l}0.20 \\
-0.10\end{array}$ & $\begin{array}{r}-0.20 \\
0.08\end{array}$ & 0.13 \\
\hline Apariencia física & 0.07 & -0.03 & -0.15 & 0.04 & 0.39 \\
\hline Relaciones cercanas & -0.20 & -0.22 & -0.03 & 0.02 & 0.18 \\
\hline Competencia atlética & -0.33 & 0.12 & 0.13 & 0.08 & -0.22 \\
\hline
\end{tabular}


La administración del hogar (-0.36), así como la sociabilidad ($0.32)$ y competencia atlética (-0.33) son los dominios que muestran una diferencia significativa entre maestros y maestras, se presenta un puntaje mayor en hombres que en mujeres. De la misma manera, los dominios de autovaloración global (0.33) y moralidad (0.34) presentan un mayor puntaje en mujeres que en hombres.

Los dominios de inteligencia (-0.27), sociabilidad (-0.25) y competencia laboral $(-0.28)$ se diferencian significativamente de acuerdo con la institución que egresaron; se presenta un puntaje mayor en casi todos los dominios, a excepción de competencia atlética (0.12), en los maestros egresados de las universidades.

Respecto de las capacitaciones que han recibido los maestros, además del PLANCAD se observa que no se presentan diferencias de medias significativas. Sin embargo, si se realiza un análisis independiente, según las fuentes de capacitación, se encuentran diferencias significativas solo en el dominio de adecuación como proveedor de apoyo; se observa un mayor promedio en los maestros que no han recibido capacitaciones por parte del Ministerio de Educación además de la del PLANCAD.

Asimismo, existen diferencias significativas entre los promedios de los maestros que han recibido capacitaciones que no son del Ministerio de Educación y la percepción de la inteligencia (0.34), la autovaloración global $(0.38)$, la apariencia física $(0.39)$ y la moralidad (0.36), tienen mayores puntajes en estos dominios.

Finalmente, existen correlaciones entre la desesperanza aprendida y el rendimiento académico, el grado de satisfacción con la carrera estudiada y el grado de satisfacción con el trabajo que realizan como maestros (Cuadro 6). 


\section{Cuadro 6}

Correlación Pearson entre la desesperanza aprendida y algunas variables

\begin{tabular}{|lc|}
\hline Variables & $R$ \\
\hline Rendimiento académico & $-0.35^{* *}$ \\
Satisfacción con la carrera & 0.20 \\
Satisfacción con el trabajo & $-0.25^{*}$ \\
\hline
\end{tabular}

Nota. ${ }^{*} p<.05{ }^{* *} p<.01$.

Se observa que la desesperanza aprendida correlaciona de manera inversa y significativa con el rendimiento académico $(p<.01)$ y con la satisfacción con el trabajo a un nivel de significancia menor ( $p$ $<.05$ ). No se observaron relaciones significativas entre la desesperanza aprendida y el grado de satisfacción con la carrera.

\section{Discusión}

Respecto del objetivo general planteado, que se refiere a la descripción del autoconcepto en los diversos dominios, es importante señalar que los maestros estudiados tienden hacia una percepción alta en todas las áreas abarcadas (los dominios de crianza y sentido del humor no entraron en el análisis por no tener niveles de confiabilidad aceptables, mayor a .5). Sin embargo, al hacer un análisis diferencial, según los dominios del autoconcepto, se observó que, en comparación con los demás, los docentes tienen una mayor percepción en inteligencia, autovaloración global, administración del hogar y moralidad. Los maestros se perciben como intelectuales con una formación integral que contempla tanto lo moral como lo conceptual. De la misma manera, se perciben como buenos administradores de su hogar, lo que compatibiliza con el trabajo fuera de casa siendo un aspecto siempre presente en una muestra compuesta en su mayoría por mujeres. Toda esta visión tiene efectos en la percepción de la autovaloración global. 
Autoconcepto y desesperanza aprendida en un grupo de maestros de Lima Metropolitana

El autoconcepto en los dominios de competencia atlética, relaciones cercanas y apariencia física es alto; sin embargo, son los más bajos en comparación con los anteriores. Se observa nuevamente la identificación de la docencia como un trabajo intelectual que se contrapone a lo físico y atlético. Además, se debe tomar en consideración que la muestra estuvo conformada por pocos maestros de educación física. Por otro lado, el establecimiento de relaciones íntimas o cercanas se relaciona con las condiciones laborales; es decir, un maestro que recibe un bajo sueldo por su trabajo tiene que recurrir a otros empleos para poder sobrevivir (Tovar, 1989; Ministerio de Educación, 1993; Foro Educativo, 1998; Calidad de la educación, 1999; Tarea, 2000), lo que constituye una limitación que recorta sus posibilidades de enriquecimiento cultural, científico, académico y afectivo en el sentido que carece de tiempo para establecer relaciones cercanas con una pareja, compañeros o amigos y profundizar en ellas no se convierte en una prioridad.

Estos resultados confirman el modelo propuesto por Shavelson (Hoge, Smit y Crist, 1995) según el cual el autoconcepto es estructurado y jerarquizado en tres niveles identificables. En los dominios de Messer y Harter (1986) el autoconcepto global se ubicaría en lo alto de la jerarquía, en el medio sectores específicos de autoconcepto como inteligencia, moralidad, sociabilidad, competencia laboral y en la base las subáreas específicas del autoconcepto como administración del hogar (aunque esta se ubica en una posición central, según los resultados), adecuación como proveedor de apoyo, apariencia física, relaciones cercanas y competencia atlética.

El yo es una construcción tanto social como cognitiva. Cognitiva porque las autorrepresentaciones se organizan y dan un valor al cambio en el desarrollo normativo, enfatizando las semejanzas entre los individuos. Social porque se relaciona con los procesos socializadores y se producen diferencias entre las personas en la medida de que el yo sea favorable o desfavorable (Harter, 1999). 
En relación con la desesperanza aprendida, los maestros presentaron un bajo nivel de esta, es decir, poca desesperanza. Se ha encontrado resultados controversiales, como, por ejemplo, una marcada división de opiniones en el ítem 3 ("El futuro es difícil de asegurar pues no se sabe qué va pasar") y en el ítem 7 ("En la mayoría de los casos los problemas no dependen de uno mismo, sino de la difícil situación que uno vive"). Este tipo de respuestas puede deberse a dos situaciones: una se refiere al marco en el que se desenvuelven los maestros peruanos, situación que se viene arrastrando desde años atrás: por un lado existen dispositivos legales, pero con un grave incumplimiento sistemático de los mismos por parte de las autoridades responsables; un sindicato sin poder ni presencia efectiva; bajo nivel de sueldos; alumnos con niveles pobres de aprendizaje; gran número de alumnos por aula; ausentismo escolar; y escaso apoyo de las autoridades y padres de familia (Tovar, 1989; Ministerio de Educación, 1993; Foro Educativo, 1998; Calidad de la educación, 1999; Tarea, 2000). Por otro lado, el país actualmente está atravesando por una grave crisis social, política y económica que atenta contra la estabilidad y eso genera una incertidumbre en toda la población.

Asimismo, en la escala de desesperanza se presentó una marcada polarización hacia el desacuerdo con los ítems 5 ("Todos debemos conformarnos con lo que tenemos pues es muy difícil de conseguir mayores ingresos económicos") y 12 ("Creo que los problemas de mi escuela no podrán solucionarse"). Se observó una capacidad en los maestros para luchar contra la adversidad y no conformarse con la situación en la que viven. Si bien es cierto que reciben un bajo sueldo del Estado por su trabajo, ellos recurren a otros empleos con el fin de tener mayores ingresos, más aun si se trata de personas que se consideran intelectuales y que, en ese sentido, se colocan en un nivel diferente al de cualquier obrero o empleado. Además, el proceso de capacitación plantea al docente el desarrollo de habilidades, destrezas y conocimientos que le permiten un trabajo eficiente, caracterizado por un compromiso de cambio de actitud en sus métodos de enseñanza y aprendizaje. En tal sentido, la tarea que tiene el docente consiste en 
partir del reconocimiento de sus propias limitaciones, posibilidades y necesidades, así como de las expectativas que surgen de la investigación reflexiva con relación a sus experiencias en el aula (Ministerio de Educación, 2000).

Los resultados obtenidos, tanto en el perfil de autopercepción para adultos como en la escala de desesperanza aprendida, reflejan que el taller de capacitación ha ejercido un efecto en ambas medidas debido a las reacciones que puede generar en los maestros del Estado una evaluación, más aun si existe un miedo implícito al castigo o a la calificación final dentro del proceso de la capacitación mismo. Esto se evidenció cuando se presentaron algunas suspicacias entre los participantes por el anonimato mismo de las pruebas. En algunos casos no quisieron escribir su nombre y en otros preguntaban, con desconfianza, si todo esta evaluación era parte de la calificación del taller, la que es promediada, en la calificación final, al terminar el año de capacitación.

En esta investigación se plantearon cuatro hipótesis que fueron confirmadas. La hipótesis general postulaba que existen correlaciones inversas entre los distintos dominios del autoconcepto y la desesperanza aprendida en los maestros capacitados por el PLANCAD; lo que quiere decir que si un maestro capacitado tiene un nivel bajo de desesperanza aprendida, su percepción en los dominios sobre su autoconcepto serán mayores y viceversa.

El autoconcepto es una variable que organiza, interpreta y da significado a las experiencias; regula el afecto y motiva la acción dando incentivos, planes estándares y guiones; también organiza el comportamiento y energetiza al individuo para seguir metas seleccionadas (Harter, 1999). Por otro lado, la desesperanza aprendida se refiere a la atribución que la persona hace respecto de la falta de control sobre los resultados de sus respuestas; si la atribución implica factores internos al sujeto, la teoría predice la aparición de una baja en el sentimiento de autoestima de la persona (Castrillo, 1986). Es 
así que un buen autoconcepto y una baja desesperanza aprendida en los maestros debe ser un factor que favorezca al proceso educativo hacia la dirección deseada.

Sin embargo, solo algunos dominios del autoconcepto se correlacionan, de manera significativa, con la desesperanza aprendida con diferentes niveles de significancia: Los más importantes son los dominios de inteligencia y competencia laboral. Las percepciones en ambos aspectos podrían determinar, en cierta manera, su desempeño en la capacitación y en su trabajo; de esa manera se corroboraría, - de acuerdo con los resultados de diversos estudios - que no solo hay una relación consistente y moderadamente fuerte entre autoconcepto y habilidad académica, sino que estas dos variables son altamente interactivas y recíprocas (Hamachek, 1995).

De la misma manera, una baja desesperanza aprendida se relaciona inversa y significativamente con los dominios de sociabilidad, relaciones cercanas, adecuación como proveedor de apoyo y apariencia física. Al sentir mayor control sobre las respuestas, los maestros pueden manejar mejor los aspectos relacionados con estos dominios y viceversa. Además, la naturaleza misma de la muestra indica que el maestro, por tener una carrera orientada hacia el servicio de otras personas, necesita desarrollar los aspectos relacionados con ser sociable, establecer relaciones cercanas y percibirse con una apariencia física adecuada. Por otro lado, puede sentirse un adecuado proveedor de apoyo cuando siente este control sobre las consecuencias de sus acciones.

La primera hipótesis específica plantea que existen correlaciones entre los distintos dominios del autoconcepto y las variables edad, grado de satisfacción con la carrera estudiada, grado de satisfacción con el trabajo que realiza como docente y el rendimiento académico en el taller de capacitación.

Respecto de la edad, esta correlaciona positiva y significativamente con sociabilidad y relaciones cercanas, es decir, a mayor edad, 
Autoconcepto y desesperanza aprendida en un grupo de maestros de Lima Metropolitana

mayor promedio en la percepción de uno mismo en estas áreas. Las condiciones de supervivencia llevan a los maestros a asumir múltiples obligaciones en su tiempo libre (trabajar en otros empleos) (Tovar, 1989; Ministerio de Educación, 1993; Foro Educativo, 1998; Calidad de la educación, 1999; Tarea 2000). Esto ocasiona que los docentes no tengan tiempo para priorizar el establecimiento de relaciones cercanas o íntimas con amigos, compañeros o con una pareja. Esta situación mejora a mayor edad, cuando quizá no apremia tanto lo económico y se haya logrado mayor estabilidad, los hijos son mayores, trabajan y quizá hasta reciba un sueldo de jubilación.

Otro aspecto a discutir se refiere al grado de satisfacción que los maestros sienten por la carrera estudiada y el trabajo realizado (entre lo ideal y lo real), las cuales correlacionan significativamente con la competencia laboral, adecuación como proveedor de apoyo y relaciones cercanas. Es decir, mientras más satisfechos se sientan los maestros con lo que han estudiado y cómo están trabajando, mayor será el promedio en su percepción en el área laboral, podrán sentirse más adecuados como proveedores de apoyo para su familia y sentirán mayor capacidad para establecer relaciones cercanas.

De la misma manera, el grado de satisfacción con la carrera estudiada correlaciona significativamente con el dominio de sociabilidad y de manera inversa con la competencia atlética. Se observa nuevamente la identificación de la docencia como una carrera intelectual, de servicio a los demás, que se contrapone a lo atlético; mientras que el grado de satisfacción con el trabajo presenta una relación con inteligencia, administración del hogar y apariencia física porque el tener un trabajo fuera de casa, reconocido como intelectual, implica percibirse con cierto nivel de inteligencia y con una adecuada apariencia física.

Este resultado se relaciona con un estudio realizado por Morante (1989). Este plantea que las madres de familia que han decidido trabajar, aun teniendo niños en edad preescolar, lo han hecho justamente porque su nivel de autoestima previo era lo suficientemente elevado 
como para tomar esa decisión. En contraposición, una persona con baja autoestima es susceptible a las críticas y se deja llevar por lo que dicen los demás. Lo que se espera con tales características, por más que tenga actitudes favorables hacia el rol del trabajo, si no es apoyada y, más aun, si es criticada por sus familiares o amigos por su deseo de salir a trabajar teniendo hijos pequeños, no será lo suficientemente fuerte como para actuar de acuerdo a sus preferencias. "El trabajo realizado puede ser una fuente de autoestima, a su vez la autoestima puede facilitar o no la opción de trabajo" (Morante, 1989).

Un aporte importante de la investigación es la relación que existe entre el autoconcepto y el rendimiento académico en la capacitación. del PLANCAD. Este correlaciona significativamente solo con el dominio de sociabilidad, es decir, a mayor sociabilidad, mayor rendimiento.

Los talleres de capacitación se desarrollan básicamente con dinámicas participativas grupales para trabajar los contenidos y las técnicas pedagógicas. En los informes elaborados al finalizar el taller, los entes ejecutores consideran como logros que los maestros hayan dialogado e intercambiado experiencias, compartido resultados, expuesto sus trabajos, socializado entre ellos, que hayan leído en grupo, participado en dinámicas, identificado y compartido sus logros y que hayan propuesto alternativas de solución frente a las dificultades que se les presentan en su quehacer pedagógico. Los maestros con alta percepción de su sociabilidad podrían responder a estas exigencias. Por otro lado, los capacitadores que dirigen este taller podrían también estar prestando mayor atención à los maestros más participativos y extrovertidos generando un efecto por ambos lados (del capacitado y del capacitador). Las expectativas de los que enseñan pueden tener gran influencia sobre el grupo, principalmente en la evaluación.

Harter (1999) plantea que los estudiantes necesitan de estímulos y apoyo para expresar sus opiniones, tanto de los maestros como de sus compañeros de clase, porque necesitan ser escuchados. Sin embargo, para que los estudiantes puedan expresarse por sí solos, necesitan 
oportunidades educacionales. Situaciones en lo que los maestros simplemente "instruyen", transmitiendo los conocimientos para que sean aprendidos por el grupo, apoyan poco las intervenciones de los estudiantes. Por otro lado, los educadores, dentro de un movimiento del aprendizaje cooperativo, dan a los estudiantes la oportunidad de exponer sus ideas en un contexto en el que pueden ser escuchados y entendidos.

La segunda hipótesis específica plantea que existen diferencias significativas entre los distintos dominios del autoconcepto según las variables sexo, institución en la que el docente terminó sus estudios y otras capacitaciones recibidas además del PLANCAD.

En este sentido, se puede ver que los hombres presentan una mayor autopercepción en cómo administran su hogar, su capacidad para socializar y sus habilidades atléticas mientras que las mujeres destacan por una mejor percepción en su autovaloración global y su moralidad. Las mujeres que trabajan pueden ser más autocríticas en su rol tradicional de amas de casa y percibir que no están cumpliendo con lo esperado; más aun, si se trata de mujeres que tienen familia a su cargo (esposo e hijos).

Con frecuencia, el sistema educativo de los países latinoamericanos ha resultado discriminador en cuanto al género (Bulnes, 1999). Por ejemplo, los textos de la educación primaria presentan en sus imágenes una visión bastante estereotipada del mundo social: las mujeres restringidas al espacio doméstico realizando labores tradicionalmente femeninas y los varones enfrentados a un mundo de trabajo organizando la sociedad. Existen estudios (Bulnes, 1999) que demuestran que en los materiales bilingües del Ministerio de Educación (DINEBI) se presenta una ausencia casi total de un adecuado tratamiento del género, eso indica que existe una considerable brecha entre lo que predicen y lo que hacen (la teoría y la práctica). Esto hace suponer que los maestros no han terminado de comprender la real dimensión de este tema, se siguen presentando los papeles tradicionales 
de la mujer en desmedro de su pleno desarrollo intelectual y de su indispensable participación social.

Con relación a una mejor autovaloración global o autoestima, cabe destacar que la situación laboral de las mujeres, que son madres, influye en su actitud hacia los diferentes roles en la autoestima; debe considerarse que el nivel de este aspecto también puede conducir a la madre a optar por determinada situación laboral que puede o no ser de su preferencia. Cuanto más elevada es la autoestima de la mujer, menos tradicionales son sus preferencias por una carrera y la elección realista del trabajo (Morante, 1989).

Estos resultados pueden ser analizados desde la perspectiva del estudio desarrollado por Block y Robins (Harter, 1999); en él se plantea que la habilidad de relacionarse con otros, en una manera positiva interpersonalmente, promueve la autoestima en las mujeres; por otro lado, ser independientes, sin involucrarse emocionalmente con otros, está altamente relacionado con la autoestima en los hombres.

Por otro lado, Miller (Harter, 1999) plantea que siempre ha habido una dicotomía entre las orientaciones "centradas en el self" - self-focused-, es decir, un estilo más individualista asociado a los hombres. Las orientaciones "centradas en el otro" (other-focused) —estilo más conectado hacia las otras personas- usualmente es atribuido a las mujeres. De acuerdo con esta tipología, los individuos focalizados en el self están caracterizados como excesivamente autónomos, independientes y dominantes. En contraste, los individuos centrados en los otros están orientados a cuidar y preocuparse por los demás, así como ser condescendientes y subordinar sus propias necesidades a las de los demás.

Con relación al tipo de institución en que los maestros terminaron su carrera, se observaron diferencias significativas respecto de los dominios de inteligencia, sociabilidad y competencia laboral; se presenta un mayor promedio en la percepción en los egresados de las universidades en comparación a los egresados de institutos, quienes 
se consideran más hábiles en lo atlético. La universidad como institución presenta cierto estatus que se refleja en la confianza y aceptación social. Es probable también que exista una sobrevaloración de esta en la sociedad peruana misma. Por otro lado, valdría la pena cuestionarse si la formación docente en institutos superiores pedagógicos, privados y estatales, no está dando suficientes elementos teóricos y prácticos para que los egresados se sientan mejor preparados.

Otro aporte interesante de esta investigación lo constituye las diferencias que se presentan entre los dominios del autoconcepto y las capacitaciones pedagógicas recibidas, además de la del PLANCAD. Se observó que no se presentan diferencias significativas en los dominios del autoconcepto según las capacitaciones en general; sin embargo, si se y analizan independientemente las fuentes de capacitación, se encuentran diferencias significativas en el dominio de adecuación como proveedor de apoyo en tanto se presenta un mayor promedio en los maestros que no han recibido capacitaciones por parte del Ministerio de Educación. Los que han recibido otras capacitaciones no otorgadas por el Ministerio de Educación presentan diferencias significativas en los dominios de inteligencia, autovaloración global, moralidad y apariencia física.

Una posible explicación de estos resultados podría ser que las capacitaciones del Ministerio de Educación se perciben como obligatorias para cierto grupo de docentes (a pesar de no serlo explícitamente, en muchos casos). Todo esto está inmerso en el marco de la normatividad y de los reglamentos, quizá por el temor a no perder su plaza o estar desactualizados con las nuevas normas. Además, podría tratarse de factores relacionados con la institución misma del gobierno y la gratuidad de los programas. El PLANCAD, a diferencia de las otras capacitaciones del Ministerio de Educación, se presenta como un programa más que un conjunto de talleres. Por otro lado, en las capacitaciones que no son del Ministerio de Educación sucedería lo contrario, ya que pueden ser tomadas por iniciativa propia bajo sus propios 
gastos e intereses. Se observan factores motivacionales importantes que determinan el esfuerzo y el interés que influye en los resultados.

La tercera y última hipótesis específica plantea que existe una correlación inversa entre la desesperanza aprendida y el rendimiento académico en el taller de capacitación, así como con el grado de satisfacción con la carrera estudiada y el grado de satisfacción con el trabajo que realizan como docentes.

La desesperanza aprendida correlaciona de manera inversa y significativa con el rendimiento académico, es decir, a menor desesperanza aprendida, mayor rendimiento académico. Una revisión de los estudios realizados sobre la primera y el éxito académico (Mark, 1978) planteó que se obtienen diferentes resultados cuando los indicadores son comportamentales: los sujetos saben que sus respuestas son esperadas y son consistentes con sus roles de género. Por otro lado, Hayes (1979) plantea que las experiencias del éxito en las personas, que están en un proceso de aprendizaje, son importantes, así como el desarrollo de mecanismos para evitar fallas eventuales.

Por otro lado, el grado de satisfacción con el trabajo correlaciona de manera inversa y significativa con la desesperanza aprendida. Esta satisfacción genera que se sienta control sobre los efectos de las propias acciones, en este caso laborales o, de manera inversa, que el control percibido genere esta satisfacción.

A modo de conclusión, toda esta investigación sugiere que no basta tener condiciones de trabajo adversas para poder desarrollar una desesperanza aprendida. Todo depende de los estilos de atribución y de cómo las personas perciben su posibilidad de controlar esa situación. En este sentido, se puede pensar que los maestros participantes han desarrollado esa capacidad de lidiar contra la adversidad. Asimismo, en los talleres de capacitación del PLANCAD, se les da a los maestros alternativas para enfrentar las dificultades, por ejemplo elaborar materiales educativos con recursos de la zona o materiales reciclables. 
Autoconcepto y desesperanza aprendida en un grupo de maestros de Lima Metropolitana

Asimismo, los maestros del estudio tienen una alta autovaloración global, se perciben con niveles de inteligencia importantes, así como aspectos de la moralidad, sociabilidad y competencia laboral positivos.

Por otro lado, siendo la docencia una profesión con vocación de servicio, surge la pregunta ¿hasta qué punto esa creencia y la sensación de tener el poder para "cambiar" algo a través del proceso educativo está llevando a que los maestros siempre se sientan capaces de controlar las situaciones adversas?

La misión del maestro es lograr que los mismos estudiantes aprendan a enfrentar las situaciones difíciles, lo que genera una responsabilidad en ellos para formar a los peruanos del futuro. Esto sería una posible explicación del porqué hay una baja desesperanza.

Estos resultados son sumamente importantes porque van en contra de lo que muchas personas podrían pensar de los maestros como personas desmotivadas, desesperanzadas, inconformes y resignadas por la situación misma en la que viven y trabajan.

De alguna manera, los resultados presentados en esta investigación reivindican el rol del maestro mostrándolo como una persona que siempre está luchando contra la adversidad de la situación social, política y económica. Por otro lado, es una motivación para él pensar que su trabajo está al servicio de su país y que esto le da una tarea importante en la mejora del mismo como parte de su responsabilidad.

El encontrar niveles altos en los dominios del autoconcepto y no identificar niveles de desesperanza aprendida significativos en los maestros es un aspecto positivo porque su misión es generar el cambio a través de su quehacer y, en ese sentido, tiene que enfrentar la adversidad. La percepción de no control en este caso no tiene sentido porque su trabajo consiste en cambiar lo que no es correcto. Existiría una sensación de ser un agente con el poder de generar cambios para el futuro. 
Si bien es cierto que se podría traducir en una especie de "omnipotencia docente", también se puede interpretar como un rol que la sociedad misma le atribuye. Por ejemplo, los padres mandan a sus hijos a la escuela para que aprendan y sean mejores, tengan una profesión y no sean ignorantes, de tal modo que delegan a los maestros esta responsabilidad. De la misma manera, los maestros son portadores de cultura y superación, lo que se complementa con su percepción en los dominios de inteligencia, autovaloración global, moralidad y competencia laboral. La visión integral del nuevo enfoque pedagógico plantea que la educación debe abarcar tanto los aspectos conceptuales, procedimentales y actitudinales como también los valorativos relacionados con la moral.

Por otro lado, la docencia, sobre todo en los primeros grados de primaria, es una profesión casi exclusiva del género femenino y en ese sentido se debe tener cuidado respecto de los roles que en esta se genera cuando son tradicionales. Es por ello que las maestras sienten una percepción más baja en la manera cómo administran su hogar en comparación con los maestros y estos se perciben mejores en lo social y atlético. Se observan diferencias de género en donde las actividades del espacio privado corresponden a la mujer mientras que las actividades del espacio publico, 'al hombre. Se debe estar alerta en este aspecto si es que se está apuntando a una reforma educativa en donde la democracia e igualdad para todos sea una característica del mismo sistema.

Asimismo, es importante señalar las diferencias encontradas en los dominios de inteligencia y competencia laboral entre los egresados de institutos superiores pedagógicos y universidades. Esto podría deberse a una mayor aceptación de la universidad en nuestra sociedad o a una formación deficiente en los institutos superiores con relación a determinados aspectos teóricos y prácticos, eso daría una sensación de no contar con algunos elementos necesarios para la labor educativa. 
Tener maestros con estas características es una ventaja para la reforma que se está realizando en el ámbito educativo en nuestro país actualmente $y$, en este sentido, esto debe ser utilizado para mejorar $y$ potencializar los cambios que se están planteando dentro de este proceso. No se puede seguir pensando en un maestro como receptor pasivo y sin recursos. Es importante tomar en serio el rol que se le adjunta como principal gestor, agente y guía del proceso educativo.

Finalmente, y como se planteó en un principio, conocer estos resultados, que describen ciertos aspectos de un grupo de maestros en cuanto a cómo ellos mismos se conciben y cómo enfrentan las situaciones del medio puede ser un inicio para futuras investigaciones que sirvan de base para enfocar la capacitación y la profesionalización docente de una manera más directa, concreta y efectiva.

Del trabajo presentado se desprenden las siguientes conclusiones:

- Los maestros tienen una percepción alta en autoconcepto. Los puntajes mayores son los de inteligencia, autovaloración global, administración del hogar y moralidad; los más bajos están en competencia atlética, relaciones cercanas y apariencia física.

- Los maestros presentan un bajo nivel de desesperanza aprendida, aunque existen diferencias en algunos aspectos, como una marcada división de opiniones en la inseguridad frente al futuro y en el grado de responsabilidad con los problemas que se presentan; por otro lado, se presentó una marcada polarización hacia el desacuerdo con las afirmaciones referidas a conformarse con lo que se tiene porque es difícil asegurarse mayores ingresos económicos y la no posibilidad de solucionar los problemas de la escuela.

- Existen correlaciones inversas entre los distintos dominios del autoconcepto y la desesperanza aprendida en los maestros capacitados por el PLANCAD. Autoconcepto de inteligencia y competencia laboral correlacionan de manera inversa y muy significativa con desesperanza aprendida. Esta correlaciona a un nivel de significancia menor $y$, de manera inversa, con autoconcepto 
de sociabilidad, relaciones cercanas, adecuación como proveedor de apoyo y apariencia física.

- Los hombres presentan una mayor autopercepción en la manera cómo administran el hogar, su capacidad para socializar y sus habilidades atléticas, mientras que las mujeres destacan por una mejor percepción en su autovaloración global y su moralidad.

- La edad correlaciona significativamente con autoconcepto de sociabilidad y relaciones cercanas.

- Se presenta un mayor promedio significativo en los egresados de universidades que los de institutos respecto de autoconcepto de inteligencia, sociabilidad y competencia laboral.

- La satisfacción por la carrera correlaciona significativamente con el autoconcepto de sociabilidad. Asimismo, la satisfacción por la carrera y el trabajo correlaciona significativamente con autoconcepto de competencia laboral, adecuación como proveedor de apoyo y relaciones cercanas.

- El grado de satisfacción con el trabajo se relaciona con los dominios del autoconcepto de inteligencia, administración del hogar y apariencia física.

- Los maestros que han recibido capacitaciones pedagógicas, además del PLANCAD, no presentan diferencias significativas en los dominios del autoconcepto; sin embargo, los que no han recibido capacitaciones del Ministerio de Educación presentan mayor promedio en adecuación como proveedor de apoyo respecto de los que sí la recibieron. Por otro lado, los maestros que tuvieron otras capacitaciones presentan diferencias significativas en autoconcepto de inteligencia, autovaloración global, moralidad y apariencia física.

- El rendimiento académico en el taller correlaciona significativamente con el dominio del autoconcepto de sociabilidad.

- La desesperanza aprendida correlaciona de manera inversa y significativa con el rendimiento académico y el grado de satisfacción con el trabajo. 
Autoconcepto y desesperanza aprendida en un grupo de maestros de Lima Metropolitana

Para futuras investigaciones se sugiere:

- Evaluar a los maestros en un ambiente de capacitación que no sea oficial. La deseabilidad social y la reacción de ser evaluados se acentúan en este tipo de situaciones.

- Desarrollar indicadores para profundizar en las razones por las que se presentan diferencias en el autoconcepto según las capacitaciones pedagógicas recibidas.

- Indagar por qué sociabilidad correlaciona con el rendimiento académico y no inteligencia o competencia laboral, como podría esperarse. Quizá, observaciones del desarrollo del taller de capacitación podrían llevar a conclusiones más válidas.

- Realizar investigaciones en diferentes grupos de maestros (de colegios particulares y del Estado, de Lima y de provincias, de primaria y secundaria, capacitados por el PLANCAD y no capacitados) para poder comparar resultados.

- Realizar posteriores investigaciones con el perfil de autopercepción para adultos con el fin de establecer relaciones con otras variables psicológicas importantes como locus de control, estilos atribucionales, autoeficacia y motivación de logro, entre otras.

\section{Referencias}

Arul, M. J. (1972). A study of self concept related to adjustment and achievement. Sinopsis de la disertación enviada a la Facultad de Educación y Psicología de la Universidad de Baroda, requisito para optar el grado de Master en Psicología (En línea). Disponible: http://www.geocities.com/Athens/5503/selfcon.html.

Bulnes, M. (1999). Reflexiones, criterios y recomendaciones sobre códigos visuales, género e interculturalidad en textos para la educación intercultural bilingüe. Informe no publicado de la consultoría realizada para el PLANCAD/GTZ, Ministerio de Educación.

Calidad de la Educación ha descendido a niveles críticos (1999, 2 de Agosto). La República, p.14, 15. 
Carrillo, S. (2000). Autoconcepto y Desesperanza Aprendida en un grupo de maestros de Lima Metropolitana. Tesis para optar por el título de Licenciatura en Psicología, Pontificia Universidad Católica del Perú, Lima.

Castrillo, J. (1986). Investigación sobre la teoría de la desesperanza aprendida. Replicación de un experimento con estudiantes universitarios peruanos. Tesis para optar el grado de Bachiller en Psicología, Pontificia Universidad Católica del Perú, Lima.

Escurra, L. (1988). Cuantificación de la validez de contenido por criterio de jueces. Revista de Psicología de la Pontificia Universidad Católica del Perú, 6 (1 y 2), 103-111.

Foro Educativo (1998). Informe mundial sobre la educación: los docentes en la mira. Contacto Foro, 16, 1.

Hamachek, D. (1995). Self-concept and school achievement: Interaction dynamics and a tool for assessing the self concept component. Journal of Counseling \& Development, 73 (4), 419-425.

Harter, S. (1999). The Construction of the Self. Nueva York: The Guilford Press.

Hayes, C. (1979). Learned Helplessness and academic failure. Abstract. En línea: http://askeric.org/plweb-cgi/fastweb?search.

Hoge D., Smit, E. y Crist, J. (1995). Reciprocal effects of self-concept and academic achievement in sixth and seventh grade. Journal of Youth \& Adolescence, 24 (3), 295-314.

Macassi, S. (1988). Tipos de socialización y desesperanza aprendida. Revista de Psicología de la PUCP, 6 (1 y 2), 117-129.

Mark, S. (1978). To succeed or not to succeed: is that the only question in learned helplessness? A critical analysis of theoretical and methodological issues. Abstract de: Archivo en línea: http:// askeric.org/plweb-cgi/fastweb?search.

Messer, B. y Harter, S. (1986). Manual for the Adult Self Perception Profile. Colorado: University of Denver.

Ministerio de Educación (1993). Diagnóstico General de la Educación. Procesos pedagógicos, renumeraciones y condiciones del trabajo docente. Lima: Instituto de Pedagogía Popular. 
Autoconcepto y desesperanza aprendida en un gnupo de maestros de Lima Metropolitana

Ministerio de Educación (1999). Los eventos de capacitación de docentes en el marco del PLANCAD. Orientaciones conceptuales a los entes ejecutores. Lima: autor.

Ministerio de Educación (2000). Manual para docentes de educación primaria. Plan Nacional de Capacitación Docente PLANCAD. Lima: autor.

Morante, A. (1989). Actitudes hacia el rol del trabajo y del hogar en madres trabajadoras y no trabajadoras y su relación con la autoestima. Tesis para optar por el grado de Bachiller en Psicología, Pontificia Universidad Católica del Perú, Lima.

Tarea (2000). La docencia revalorada. Perspectivas y propuestas para el desarrollo magisterial. Lima: autor.

Tovar, T. (1989). Ser Maestro. Condiciones de Trabajo Docente en el Perú. Lima: DESCO. 